\title{
Avaliação de métodos de correção do estande para estimar a produtividade em milho(1)
}

\author{
Edilson Romais Schmildt(2), Cosme Damião Cruz $^{(3)}$, José Cola Zanuncio ${ }^{(3)}$, \\ Paulo Roberto Gomes Pereira( ${ }^{(3)}$ e Romário Gava Ferrão(4)
}

\begin{abstract}
Resumo - Este trabalho objetivou determinar o método de correção de estande mais adequado aos dados de produtividade na cultura do milho, na avaliação de 33 cultivares, em oito ambientes. Os métodos avaliados foram: sem correção, regra de três, método de Zuber, covariância de estande médio, covariância de estande ideal, o método proposto por Cruz, o método proposto por Vencovsky \& Cruz, e o proposto neste trabalho, de correção estratificada com base no agrupamento de cultivares, para a característica estande, pelo teste de Scott \& Knott. O método proposto neste trabalho, o de covariância de estande ideal e o de Vencovsky \& Cruz foram mais adequados para essa correção, pois apresentaram baixos valores de coeficiente de variação e altos valores de $\mathrm{F}$, confirmados pelos baixos valores de $\mathrm{P}_{\mathrm{i}}$ e $\mathrm{P}_{\mathrm{i}}$ multivariado na análise conjunta. Os métodos de regra de três e o proposto por Cruz apresentaram as maiores médias de produção, o que superestima o valor real da produção média. O método proposto neste trabalho foi eficiente em corrigir as cultivares, uma vez que as cultivares mais produtivas foram também as de maior potencial em deixar descendentes, medido pelo estande médio.
\end{abstract}

Termos para indexação: experimentação, análise de dados, análise estatística, melhoramento de população.

\section{Evaluation of stand correction methods to estimate productivity in cornfields}

\begin{abstract}
The objective of this work was to determine the method of stand correction more adequate to estimate productivity in cornfields of 33 varieties in eight situations. The methods tested were: without correction, rules of three, method of Zuber, covariance of medium stand, covariance of ideal stand, proposed by Cruz, proposed by Vencovsky \& Cruz, the method proposed in this work, which consisted of a stratified correction based on the combined productivity for the characteristic stand, by Scott Knott's test. The methods proposed in this work, covariance of ideal stand and that proposed by Vencovsky \& Cruz were considered the most appropriate ones, because they presented lower values for the coefficient of variation and high value of $F$, which was confirmed by the low values of $P_{i}$ and $P_{i}$ multivariate in the combined analysis. The methods rules of three and proposed by Cruz showed the highest averages of productivity, which overestimate the real value of the average production. The method proposed in this work was efficient to correct the productivity of the corn varieties, once the most productive varieties were also those with higher potential to leave descendants, measured by the average stand.
\end{abstract}

Index terms: experimentation, data analysis, statistical analysis, stand improvement.

(1) Aceito para publicação em 9 de outubro de 2000.

Extraído da Tese de Doutorado, apresentada pelo primeiro autor à Universidade Federal de Viçosa (UFV), Viçosa, MG

(2) Universidade Federal do Espírito Santo, Centro Agropecuário, Dep. de Fitotecnia, Caixa Postal 16, CEP 29500-000 Alegre, ES. E-mail: edilson@npd.ufes.br

${ }^{(3)}$ Universidade Federal de Viçosa, Dep. de Biologia Geral CEP 36571-000 Viçosa, MG. E-mail: cdcruz@mail.ufv.br, zanuncio@mail.ufv.br, ppereira@mail.ufv.br

(4) Instituto Capixaba de Pesquisa, Assistência Técnica e Extensão Rural, Caixa Postal 62, CEP 29900-970 Linhares, ES E-mail: crdrlinhares@incaper.es.gov.br

\section{Introdução}

A desuniformidade de estandes em unidades experimentais é um dos problemas básicos na análise e interpretação de resultados experimentais. Por isto tenta-se obter estandes uniformes pela semeadura em excesso, efetuando-se o desbaste para o estande desejado quando as plantas estão em estádio inicial de crescimento. Entretanto, mesmo com essa prática, a parcela pode apresentar falhas por fatores que fogem do controle do pesquisador como elevada precipitação 
pluvial, ação de pragas e doenças, o que pode afetar a realização de certas análises estatísticas, em particular quando o caráter não é medido, apenas, nas plantas competitivas (Vencovsky \& Cruz, 1991).

Estudos comparativos de rendimento de milho têm demonstrado que a proporcionalidade linear entre o número de plantas e o rendimento observado não existe. Assim, o ajuste do rendimento, para o estande ideal, por regra de três simples, constitui um procedimento inadequado, que pode superestimar o rendimento de diferentes tratamentos (Ávila \& Sánches, 1978; Vencovsky \& Cruz, 1991; Veronesi et al., 1995). Por isso, uma relação mais precisa deve levar em consideração tanto o decréscimo na produção pela presença de falhas como o acréscimo pelas plantas vizinhas às falhas, pela ausência de competição (Vencovsky \& Cruz, 1991).

Entre algumas técnicas de ajuste, em ensaios de milho, a fórmula de Zuber (1942) acrescenta 70\% do rendimento médio por planta, para cada falha, e considera que $30 \%$ são recuperadas pelas plantas que não falharam. Apesar de ser largamente utilizada por melhoristas, a proposta de Zuber (1942) tem sido criticada por não levar em consideração, no ajuste, a disposição de falhas no campo e o coeficiente de compensação pela falta de competição, que é considerado igual a 0,3 . Isto poderá ser alterado quando se avaliam tratamentos genéticos, com diferentes estruturas genotípicas, ou se conduzem ensaios em condições ambientais variadas (Veronesi et al., 1995).

Morais et al. (1986b) verificaram acréscimos de $24,5 \%$ e $32,5 \%$ na produção das plantas vizinhas às falhas devido à ausência de competição, nas cultivares Ag-7071 e Ag-301, respectivamente, o que evidencia a capacidade de recuperação da produção dos genótipos em presença de falhas. Esses mesmos autores, em ensaios de produção com a cultivar de milho BR-200, estimaram coeficientes de compensação de 0,358 e 0,156 nos anos agrícolas de 1982/83 e 1983/84, respectivamente, concluindo que a recomendação de uma fórmula com um fator único para a correção da produção de milho, como proposto por Zuber (1942), não é viável. Isto foi também encontrado por Veronesi et al. (1995), com 49 cultivares analisadas em 19 ambientes, cujos coeficientes de compensação variaram de 0,256 a 1,097, demonstrando que também o ambiente influencia a resposta das cultivares.
Morais et al. (1986a) relataram que a correção por análise de covariância, corrigindo-se a produção de grãos de milho para o estande ideal, e a correção pelo fator de correção médio, parecem ser mais indicados para a correção da produção de grãos de milho.

Ávila \& Sánches (1978) concluíram que a correção de rendimento de sorgo por fórmulas como a de Zuber (1942), utilizando um coeficiente de compensação estimado no ensaio, e aquelas com ajuste pela análise da covariância foram as mais eficientes.

Vencovsky \& Cruz (1991) concluíram que o método de correção da produção de parcelas com falhas por covariância com extrapolação para o estande ideal foi o mais eficiente em qualquer situação (níveis de falha e diferentes precisões experimentais). O método proposto por esses pesquisadores, baseado em um fator de compensação estimado dos dados, também se mostrou vantajoso, por fornecer uma medida confiável da capacidade de cultivares em recuperar o rendimento perdido pela morte de plantas. A eficiência desses métodos foi, também, comprovada para dados reais de campo por Veronesi et al. (1995), os quais mostraram que os métodos da covariância para o estande ideal e o proposto por Vencovsky \& Cruz (1991) foram os mais eficientes para a cultura do milho.

Segundo Steel \& Torrie (1980), a correção deve ser empregada apenas quando as diferenças entre tratamentos, para o estande final por parcela, forem nãosignificativas. No entanto, tem-se realizado a correção em todos os ambientes, utilizando-se a covariância para estande ideal, por permitir a estabilização dos valores ajustados ao redor do estande ideal, que deve ser comum a todos os ensaios (Vencovsky \& Cruz, 1991; Veronesi et al., 1995).

Considerando a existência de diferença estatística pelo teste $\mathrm{F}$, para as médias dos genótipos com relação ao estande final por parcela, a recomendação de não se fazer a correção do rendimento parece não ser totalmente apropriada, porém entende-se que não é adequado fazer-se a correção do rendimento das parcelas por um valor fixo do coeficiente de regressão residual. Neste caso, considera-se que o acréscimo por planta perdida, na realidade, não deverá ser necessariamente o mesmo para todas as cultivares, considerando que as que não possuam habilida- 
de para se ter o estande ideal, também poderiam não possuir o mesmo potencial para produtividade.

O objetivo deste trabalho foi propor um método estratificado de correção de estande, comparando sua eficiência com a dos métodos existentes, para estimar a produtividade na cultura do milho, em ambientes isolados e na análise conjunta dos mesmos.

\section{Material e Métodos}

Foram usados neste trabalho dados obtidos no Espírito Santo, pelo Instituto Capixaba de Pesquisa, Assistência Técnica e Extensão Rural (INCAPER), e em Minas Gerais, pela Universidade Federal de Viçosa (UFV), referentes a ensaios regionais de competição de milho precoce (Zea mays L.), safra agrícola 1996/97. Os dados originais referem-se à produção de grãos, em kg/parcela.

Foram utilizadas 33 cultivares avaliadas em blocos casualizados, com três repetições, em oito ensaios. Em todos os ensaios, as parcelas experimentais constaram de duas fileiras de 5,0 m, com espaços, entre si, de $0,90 \mathrm{~m}$, com área total por parcela de $9 \mathrm{~m}^{2}$.

O plantio foi realizado nos meses de outubro de 1996 (Sooretama, Linhares, Cachoeiro, Coimbra e Coimbra, caracterizando os ambientes 1, 2, 3, 7 e 8, respectivamente) e março de 1997 (Sooretama, Cachoeiro e Linhares, caracterizando os ambientes 4,5 e 6 , respectivamente) As adubações de plantio e de cobertura foram realizadas com base nas análises de solo e os tratos culturais e fitossanitários, de acordo com a necessidade da cultura, exceto nos ensaios 7 e 8 . No ensaio 7 , não houve aplicação de qualquer tipo de inseticida durante todo o ciclo da cultura. No entanto, no ensaio 8 , houve aplicação semanal de inseticida à base de $1,25 \mathrm{~g}$ de deltametrina/ $100 \mathrm{~L}$ de água, desde a quarta semana após plantio até o florescimento (oitava semana após emergência das sementes), visando ao controle da lagarta-do-cartucho, Spodoptera frugiperda (Lepidoptera: Noctuidae).

O ajuste, ou não, do rendimento, em razão do estande variável, foi feito com oito técnicas, considerando-se, em todas elas, uma padronização prévia para teor de umidade, em 15,5\%, conforme Ávila \& Sánches (1978).

Nas expressões de correção, $Z_{\text {ij }}$ representa o rendimento corrigido, $\mathrm{e}_{\mathrm{ij}}$, o rendimento observado nas parcelas, cujo estande é de $\mathrm{X}_{\mathrm{ij}}$ plantas. Quando presente na expressão, $\mathrm{H}$ representa o estande ideal de 50 plantas em todos os ambientes. Os oito métodos utilizados são mencionados a seguir, sendo os sete primeiros os mesmos utilizados por Vencovsky \& Cruz (1991) com dados simulados e o oitavo uma proposta a ser avaliada neste trabalho: a) SC: sem correção. Os rendimentos foram submetidos à análise, desconsiderando-se a ocorrência de falhas nas parcelas:

$\mathrm{Z}_{\mathrm{ij}}=\mathrm{Y}_{\mathrm{ij}}$.

b) RT: correção por regra de três. Os rendimentos foram corrigidos pela expressão

$\mathrm{Z}_{\mathrm{ij}}=\mathrm{Y}_{\mathrm{ij}} \cdot\left(\mathrm{H} / \mathrm{X}_{\mathrm{ij}}\right)$.

c) Z: correção pela fórmula proposta por Zuber (1942), que acrescenta $70 \%$ do rendimento médio da parcela por falha, ou seja:

$\mathrm{Z}_{\mathrm{ij}}=\mathrm{Y}_{\mathrm{ij}} \cdot\left[\mathrm{H}-0,3 \cdot\left(\mathrm{H}-\mathrm{X}_{\mathrm{ij}}\right)\right] / \mathrm{X}_{\mathrm{ij}}$.

d) COVM: utilizando-se a análise de covariância, com correção para o estande médio. $\mathrm{O}$ ajuste é freqüentemente feito por um modelo linear, pressupondo-se que a variação no rendimento dos tratamentos possa ser quantificada pelo mesmo coeficiente $b$, ou seja:

$\mathrm{Z}_{\mathrm{ij}}=\mathrm{Y}_{\mathrm{ij}}-\mathrm{b} .\left(\mathrm{X}_{\mathrm{ij}}-\overline{\mathrm{X}}_{. .}\right)$

sendo que b é o coeficiente de regressão residual de $Y_{\mathrm{ij}}$ em função de $\mathrm{X}_{\mathrm{ij}}$, estimado conforme o processo descrito por Steel \& Torrie (1980), e $\bar{X}$, o estande médio do ensaio. e) COVI: correção utilizando-se a análise de covariância, com correção para o estande ideal:

$Z_{\mathrm{ij}}=\mathrm{Y}_{\mathrm{ij}}-\mathrm{b} .\left(\mathrm{X}_{\mathrm{ij}}-\mathrm{H}\right)$

a partir do qual se estima, segundo Schmildt (2000): $\mathrm{a}=(\overline{\mathrm{Y}}-\mathrm{b}) / \overline{\mathrm{Y}}$

sendo a, o coeficiente de compensação por ausência de competição. Mede a capacidade média de compensação de rendimento dos tratamentos para cada falha na parcela. O seu conhecimento é útil para indicar a necessidade de acréscimo no rendimento da parcela e para indicar o grau de recuperação do rendimento de uma lavoura quando há redução no número inicial da população (Vencovsky \& Cruz, 1991; Veronesi et al., 1995). Ressalta-se que este estimador é diferente daquele descrito por Vencovsky \& Cruz (1991), sendo operacionalmente mais simples de ser obtido por

$\overline{\mathrm{Y}}=\mathrm{Y} / \mathrm{X}$;

sendo b, obtido conforme descrito para o método COVM. f) C: correção utilizando-se a análise de covariância, segundo o modelo proposto por Cruz (1971):

$\mathrm{Z}_{\mathrm{ij}}=\mathrm{Y}_{\mathrm{ij}} \cdot\left(\mathrm{H} / \mathrm{X}_{\mathrm{ij}}\right)$ - c $\cdot\left(\mathrm{H}-\mathrm{X}_{\mathrm{ij}}\right)$

sendo que c é o coeficiente de regressão residual da variável $\mathrm{Y}_{\mathrm{ij}}$, corrigida por regra de três, em função do número de falhas na parcela.

g) VC: correção por um fator de compensação estimado a partir dos dados experimentais, segundo o modelo proposto por Vencovsky \& Cruz (1991):

$\mathrm{Z}_{\mathrm{ij}}=\mathrm{Y}_{\mathrm{ij}} \cdot\left[\mathrm{H}-\mathrm{a} \cdot\left(\mathrm{H}-\mathrm{X}_{\mathrm{ij}}\right)\right] / \mathrm{X}_{\mathrm{ij}}$ 
a partir do qual se estima o coeficiente de compensação por ausência de competição (a) segundo Vencovsky \& Cruz (1991):

$\mathrm{a}=\mathrm{c} / \overline{\mathrm{Y}}$,

sendo c o coeficiente definido no método anterior.

Neste trabalho, chamaremos de ã e â aos coeficientes de compensação por ausência de competição obtidos por Vencovsky \& Cruz (1991) e Schmildt (2000), respectivamente.

h) Método proposto - CE: correção estratificada. Baseada na correção pela análise de covariância para estande ide$\mathrm{al}$, considerando-se a obtenção de $u m b_{k}$ para cada grupo de genótipos. Estes grupos podem ser estabelecidos arbitrariamente ou por técnica de agrupamento baseada em algum critério de otimização, ou como feito neste trabalho, utilizando o método de agrupamento de Scott \& Knott (1974). Os grupos de médias foram estabelecidos apenas nos ambientes cujo teste $\mathrm{F}$ tenha sido significativo, para o estande final por parcela.

Assim sendo:

$\mathrm{Z}_{\mathrm{ijk}}=\mathrm{Y}_{\mathrm{ijk}}-\mathrm{b}_{\mathrm{k}} \cdot\left(\mathrm{X}_{\mathrm{ijk}}-\mathrm{H}\right)$

em que,

$Z_{\mathrm{ijk}}$ é o rendimento corrigido para a i-ésima cultivar na j-ésima repetição no k-ésimo grupo estabelecido com base na inexistência de diferença significativa entre as médias, conforme teste de Scott \& Knott, realizado ao nível $\alpha$ de significância;

$\mathrm{Y}_{\mathrm{ijk}}$ é o rendimento antes da correção, para a i-ésima cultivar na j-ésima repetição e no k-ésimo grupo;

$b_{k}$ é o valor de $b$ para o k-ésimo grupo $(k=1,2, \ldots n$, sendo $\mathrm{n}$ o número de grupos).

A partir da expressão acima, estima-se o coeficiente de compensação por ausência de competição para cada grupo de cultivares obtido:

$\hat{\mathrm{a}}_{\mathrm{k}}=\left(\overline{\mathrm{Y}}_{\mathrm{k}}-\mathrm{b}_{\mathrm{k}}\right) / \overline{\mathrm{Y}}_{\mathrm{k}}$.

Nas avaliações feitas, inicialmente realizou-se a análise de variância do estande final.

Nas comparações da eficiência relativa das técnicas de correção de estandes, em cada ambiente, utilizou-se como medida o coeficiente de variação experimental (CV) e os valores da estatística F calculada para testar a hipótese de diferenças estatísticas entre médias de tratamentos, conforme Veronesi et al. (1995), que admitem como melhor método de correção aquele que: 1) minimize as variações de produção devidas a estandes variados entre as parcelas de um mesmo tratamento, proporcionando, conseqüentemente, baixos valores do coeficiente de variação; 2) corrija para fatores ambientais e não altere as variações genéticas refletidas pelas diferenças entre médias de tratamentos, e assim, não provoque reduções nos valores de $\mathrm{F}$.
Nas análises individuais, determinaram-se ainda, em cada método de ajuste, as médias gerais de produção. No método VC, foram determinados os valores do coeficiente de compensação por ausência de competição (ã) para cada ambiente, para medir a associação com o â do método COVI, por meio da correlação de Pearson.

$\mathrm{Na}$ análise conjunta de ambientes foi proposta a identificação de um método de ajuste que permita a obtenção dos menores valores de coeficiente de variação e maiores de $\mathrm{F}$, constantes nos vários ambientes. Por isto foram usados, para cada estatística, isoladamente ou ambas conjuntamente, princípios da metodologia para adaptabilidade e estabilidade de Lin \& Binns (1988):

1) para cada estatística (CV ou F)

$\mathrm{P}_{\mathrm{i}}=\sum_{\mathrm{j}=1}^{\mathrm{n}}\left(\mathrm{W}_{\mathrm{ij}}-\mathrm{M}_{\mathrm{j}}\right)^{2} / 2 \mathrm{e}$

tal que,

$\mathrm{P}_{\mathrm{i}}$ é a estimativa da eficácia do método i. Quanto menor o valor de $\mathrm{P}_{\mathrm{i}}$, mais eficaz é o método;

$\mathrm{W}_{\mathrm{ij}}$ é o valor de $100 / \mathrm{CV}$ ou, $\mathrm{F}$ do i-ésimo método no j-ésimo ambiente, sendo que $\mathrm{P}_{\mathrm{i}}$ torna-se $\mathrm{P}_{\mathrm{i}(100 / \mathrm{CV})}$ e $\mathrm{P}_{\mathrm{i}(\mathrm{F})}$, respectivamente. O valor $100 / \mathrm{CV}$ foi utilizado para que se tome como melhor método o de menor $\mathrm{P}_{\mathrm{i}}$, nas duas estatísticas de comparação (CV e F);

$\mathrm{M}_{\mathrm{j}}$ é a resposta máxima de $100 / \mathrm{CV}$ para $\mathrm{P}_{\mathrm{i}(100 / \mathrm{CV})}$ ou $\mathrm{F}$ para $\mathrm{P}_{\mathrm{i}(\mathrm{F})}$ entre todos os métodos no ambiente $\mathrm{j}$;

e é o número de ambientes.

2) multivariado, conforme princípio da medida de adaptabilidade e estabilidade de comportamento de cultivares adotado por Carneiro (1998):

$\mathrm{P}_{\mathrm{mi}}=\sum_{\mathrm{k}=1}^{\mathrm{v}}\left[\mathrm{P}_{\mathrm{ik}} \frac{1}{\hat{\sigma}_{\mathrm{pk}}}\right]$

em que,

$\mathrm{P}_{\mathrm{mi}}$ é o estimador do $\mathrm{P}_{\mathrm{i}}$ multivariado para o método de ajuste i;

$\mathrm{P}_{\mathrm{ik}}$ é o estimador do $\mathrm{P}_{\mathrm{i}}$ para cada método i relativo à k-ésima variável;

$\hat{\sigma}_{\mathrm{pk}}$ é o desvio-padrão dos $\mathrm{P}_{\mathrm{i}}$ 's para a k-ésima variável.

\section{Resultados e Discussão}

$\mathrm{O}$ teste $\mathrm{F}$ foi significativo apenas para os ambientes 1, 3, 4 e 6, para o estande final por parcela (Tabela 1). Isto demonstra comportamento diferente das cultivares nos ambientes, apesar de o estande final por parcela apresentar pequena redução em relação ao estande ideal. Esta redução variou de 0,44\%, no ambiente 7 a $11,44 \%$, no 5 . Além disso, a significância ou não pelo teste $\mathrm{F}$, não depende da 
amplitude da variação em relação ao estande ideal, pois os ambientes com maior e menor redução não mostraram significância quanto a esse teste nessa característica, de forma semelhante ao coeficiente de variação experimental. Tanto o ambiente 5, com maior coeficiente de variação experimental $(12,82 \%)$, quanto os ambientes 8 e 7 , de segundo e terceiro menores coeficientes $(4,25$ e 4,37\%), não foram significativos.

As correções pelos métodos SC, RT, e C proporcionaram maiores valores do coeficiente de variação na maioria dos experimentos, o que pode ser comprovado pelos maiores valores de $\mathrm{P}_{\mathrm{i}(100 / \mathrm{CV})}$, quais sejam, 0,$975 ; 0,658 ; 0,647$, respectivamente (Tabela 2). Embora o método $\mathrm{Z}$ não tenha proporcionado o maior $\mathrm{CV}$ experimental, em nenhum dos ambientes, este também não apresenta o menor valor em nenhum dos ambientes. Este método mostrou o segundo maior valor em dois ambientes e entre o terceiro e o quinto nos demais, o que pode ser melhor interpretado pelo seu valor relativamente alto de $\mathrm{P}_{\mathrm{i}(100 / \mathrm{CV})}$ igual a 0,3093 . Nos ambientes não estratificados (2, 5, 7 e 8), cujo teste $F$ não apontou diferenças significativas entre as médias de estandes das cultivares (Tabela 1), os menores valores de CV foram do método COVI, e, conseqüentemente, do método $\mathrm{CE}$, pois tal método passa a apresentar resultados semelhantes aos do COVI, em tais ambientes (Tabela 2). Nos ambientes 1, 3, 4 e 6, cujo teste $\mathrm{F}$ apontou diferenças significativas entre as médias de estandes das cultivares, e que, portanto, houve estratificação dos ambientes, pelo teste de Scott

Tabela 1. Média, coeficiente de variação experimental, quadrados médios de cultivares e do resíduo e teste F para a variável estande final por parcela na produção de milho, em diferentes ambientes.

\begin{tabular}{|c|c|c|c|c|c|}
\hline \multirow[t]{2}{*}{ Ambiente } & \multirow[t]{2}{*}{ Média } & \multirow[t]{2}{*}{ CV (\%) } & \multicolumn{2}{|c|}{ Quadrado médio } & \multirow[t]{2}{*}{$\mathrm{F}$} \\
\hline & & & Cultivares & Resíduo & \\
\hline 1 & 47,88 & 5,45 & 16,18 & 6,81 & $2,37^{* * 1}$ \\
\hline 2 & 48,11 & 6,47 & 14,56 & 9,70 & $1,50^{\mathrm{ns}}$ \\
\hline 3 & 46,35 & 6,10 & 30,27 & 7,99 & $3,79^{* *}$ \\
\hline 4 & 49,46 & 3,92 & 9,17 & 3,77 & $2,43^{* *}$ \\
\hline 5 & 44,28 & 12,82 & 43,84 & 32,23 & $1,36^{\mathrm{ns}}$ \\
\hline 6 & 48,61 & 6,80 & 19,28 & 10,92 & $1,77^{*}$ \\
\hline 7 & 49,78 & 4,37 & 5,31 & 4,74 & $1,12^{\mathrm{ns}}$ \\
\hline 8 & 48,55 & 4,25 & 6,18 & 4,27 & $1,45^{\mathrm{ns}}$ \\
\hline
\end{tabular}

ns Não-significativo. ${ }^{*} \mathrm{e}^{* *}$ Significativo a $5 \%$ e a $1 \%$ de probabilidade, respectivamente.

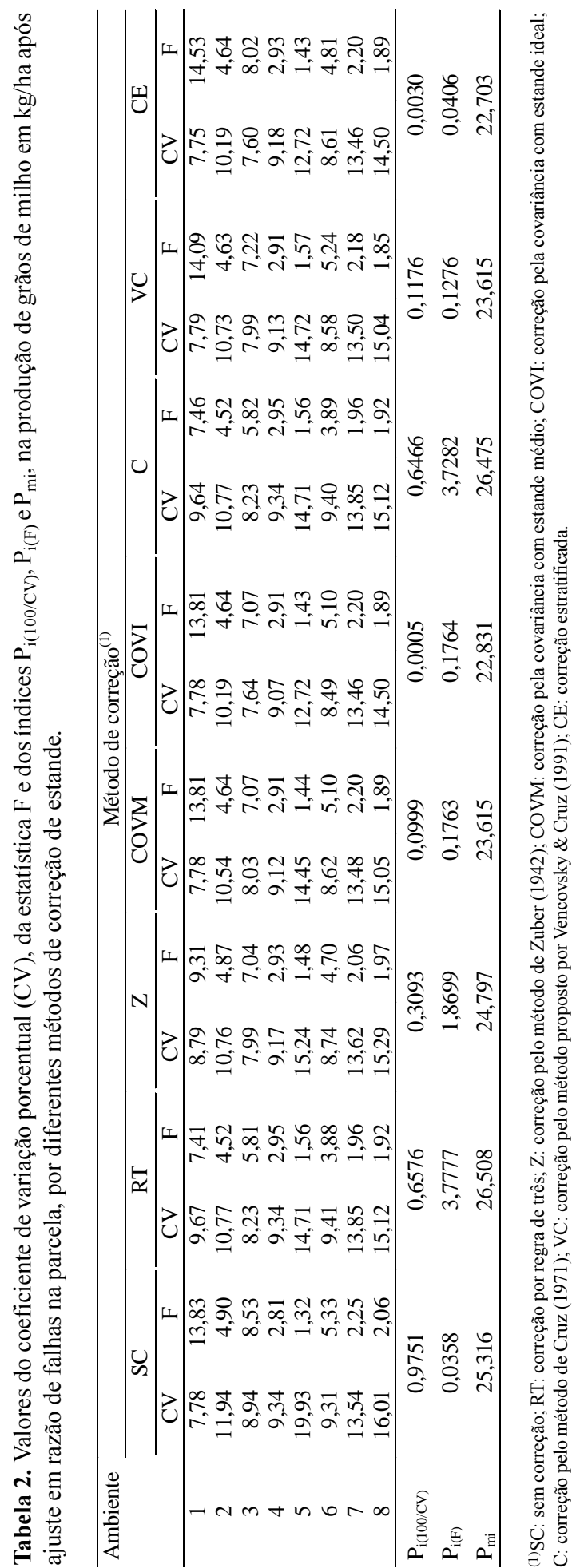

Pesq. agropec. bras., Brasília, v. 36, n. 8, p. 1011-1018, ago. 2001 
\& Knott, os menores valores de CV também ocorreram para os métodos COVI e CE, cada um sendo melhor em dois ambientes. Considerando os valores de $\mathrm{P}_{\mathrm{i}(100 / \mathrm{CV})}$, os mesmos são de 0,0005 e 0,0030 , respectivamente, nos métodos COVI e CE (Tabela 2).

Com relação aos valores de F, pode-se observar que a análise dos dados sem correção prévia proporcionou os maiores valores em cinco ambientes (Tabela 2). O método de correção baseado em regra de três proporcionou maior valor de $\mathrm{F}$ em apenas um ambiente, assim como os métodos VC e CE. Ressaltase que os métodos RT e C proporcionaram os menores valores de $\mathrm{F}$ em cinco ambientes, demonstrando a sua impropriedade para uma avaliação comparativa adequada das cultivares. Observa-se, ainda, que nos ambientes em que não foi verificado o maior valor de F, os métodos VC e CE apresentaram valores altos, próximos aos maiores observados, traduzindo-se nos baixos valores de $\mathrm{P}_{\mathrm{i}(\mathrm{F})}, 0,0406$ e 0,1276 , respectivamente, o que aponta tais métodos como os mais indicados com relação à estatística $\mathrm{F}$ (Tabela 2).

Os métodos CE, COVI, VC e COVM mostraram maior eficiência de ajuste para o valor de $\mathrm{P}_{\mathrm{mi}}$ (Tabela 2). Veronesi et al. (1995), analisando cinco diferentes métodos de ajuste em milho, mostraram que o ajuste pela covariância para estande ideal e o proposto por Vencovsky \& Cruz (1991) proporcionaram os melhores resultados em termos de adequação desse ajuste, indicados pelos baixos valores de $\mathrm{CV}$ e altos valores de F.

As médias gerais de produção em cada ambiente, independentemente de cultivares, após ajuste para cada método de correção (Tabela 3), mostram que o método RT apresentou os maiores valores em seis ambientes, e levou à superestimação dos resultados, o que o torna indesejável como relatado por Ávila \& Sánches (1978), Vencovsky \& Cruz (1991) e Veronesi et al. (1995). O método C, que utiliza o princípio da regra de três em seu ajuste, apresentou comportamento semelhante.

O método COVM proporciona valores médios de produção semelhantes ao do SC, mas apresenta o inconveniente de ter ajuste em torno do estande médio (Ávila \& Sánches, 1978). Isto provoca redução da produção das cultivares que se apresentaram com estande ideal, sendo, portanto, inconveniente como método de correção. Os demais métodos de correção permitiram obtenção de médias de produção que não se distanciaram muito do método SC, com exceção do ambiente cinco, onde o método COVI apresentou a maior média. Vencovsky \& Cruz (1991), com dados simulados, verificaram que à medida que se reduz o valor do estande médio, a média estimada de produção aumenta consideravelmente, o que pode explicar o comportamento apresentado no ambiente cinco, com a menor média do estande entre todos os ambientes (Tabela 1).

Considerando as médias de produção após cada método de ajuste (Tabela 3), apenas nos ambientes cujo teste $\mathrm{F}$ tenha sido significativo para o estande final por parcela (Tabela 1), verifica-se que os métodos COVI, VC e CE permitem obter resultados de médias bem próximas entre si, com certa distinção do método RT. Isso mostra mais uma vez a superioridade destes três primeiros métodos e, ao mesmo tempo, o comportamento não-linear do acréscimo de rendimento, pela presença de falhas, como mencionado por outros pesquisadores (Zuber, 1942; Ávila \& Sánches, 1978).

Tabela 3. Produção média ( $\mathrm{kg} / \mathrm{ha})$ de milho, em cada ambiente, independentemente de cultivares, em razão do método de correção de estande.

\begin{tabular}{ccccccccc}
\hline Ambiente & \multicolumn{9}{c}{ Método de correção $^{(1)}$} \\
\cline { 2 - 8 } & SC & RT & Z & COVM & COVI & C & VC \\
\hline 1 & 5.801 & 6.063 & 5.984 & 5.801 & 5.802 & 6.060 & 5.782 & 5.793 \\
2 & 6.511 & 6.754 & 6.681 & 6.511 & 6.733 & 6.754 & 6.736 \\
3 & 6.893 & 7.420 & 7.262 & 6.893 & 7.244 & 7.419 & 7.237 & 7.733 \\
4 & 7.329 & 7.418 & 7.391 & 7.329 & 7.370 & 7.418 & 7.375 & 7.345 \\
5 & 6.527 & 7.326 & 7.086 & 6.527 & 7.416 & 7.327 & 7.390 & 7.416 \\
6 & 7.023 & 7.232 & 7.169 & 7.023 & 7.127 & 7.231 & 7.116 \\
7 & 5.729 & 5.757 & 5.749 & 5.729 & 5.736 & 5.757 & 5.738 & 5.736 \\
8 & 5.602 & 5.762 & 5.714 & 5.602 & 5.816 & 5.763 & 5.827 \\
\hline
\end{tabular}

(1)SC: sem correção; RT: correção por regra de três; Z: correção pelo método de Zuber (1942); COVM: correção pela covariância com estande médio; COVI: correção pela covariância com estande ideal; C: correção pelo método de Cruz (1971); VC: correção pelo método proposto por Vencovsky \& Cruz (1991); CE: correção estratificada. 
A média de produção por planta, antes da correção, variou de 103,58 g no ambiente 7, a 133,85 g no 3, com variação de, apenas, $29,22 \%$ entre os ambientes (Tabela 4). No entanto, o acréscimo por planta perdida, mostrado pelo valor de b em cada ambiente, foi bastante diferenciado, variando de 0,66 a $142,11 \mathrm{~g} /$ planta, apresentando comportamento muito diferenciado entre os ambientes.

Os valores do coeficiente de compensação por ausência de competição (Tabela 4), variaram de $\hat{a}=-0,277$ a 0,994 no método COVI e de $\tilde{a}=-0,402$ a 1,072 no VC. Apenas no ambiente 3, estes valores foram próximos ao valor de 0,3 proposto por Zuber (1942). No entanto, a adequação de ajuste (Tabela 2) do método Z não supera a dos métodos COVI, VC e CE. Isto demonstra que o método proposto por Zuber (1942) não seria o mais indicado e está de acordo

Tabela 4. Produção média de milho, sem correção, coeficientes de regressão residual (b) e coeficiente de compensação por ausência de competição, em cada ambiente.

\begin{tabular}{ccccr}
\hline Ambiente & $\begin{array}{c}\text { Média de } \\
\text { produção } \\
\text { (g/planta) }\end{array}$ & $\mathrm{b}(\mathrm{g} /$ planta) & \multicolumn{2}{c}{$\begin{array}{c}\text { Coeficiente de } \\
\text { compensação }\end{array}$} \\
\cline { 4 - 5 } & 109,04 & 0,66 & 0,994 & 1,072 \\
\hline 1 & 121,81 & 105,40 & 0,135 & 0,085 \\
2 & 133,85 & 86,52 & 0,354 & 0,348 \\
3 & 133,36 & 68,69 & 0,485 & 0,471 \\
4 & 129,78 & 142,11 & $-0,095$ & $-0,096$ \\
5 & 130,03 & 67,21 & 0,483 & 0,555 \\
6 & 103,58 & 29,52 & 0,715 & 0,676 \\
7 & 103,84 & 132,56 & $-0,277$ & $-0,402$ \\
8 & & & & \\
\hline
\end{tabular}

${ }^{(1)}$ Coeficiente de compensação obtido por Schmildt (2000) a partir da análise de covariância da produção em função do estande (â) e obtido por meio do estimador descrito por Vencovsky \& Cruz (1991) (ã); coeficiente de correlação de Pearson $\left(\mathrm{r}_{(\mathrm{a}, \text { ã }}\right)$ igual a 0,9948 . com o relatado por Ávila \& Sánches (1978) e Veronesi et al. (1995).

A correlação de Pearson entre os valores de â e ã foi de 0,9948 (Tabela 4). Estes valores indicam correlação elevada entre os métodos COVI e VC, o que reforça os resultados da adequação de ajuste (Tabela 2), e corrobora com as recomendações de uso de tais metodologias por Vencovsky \& Cruz (1991) e Veronesi et al. (1995). Ressalta-se, no entanto, que, sendo â operacionalmente simples de ser obtido, recomenda-se o seu uso em substituição ao coeficiente ã que é mais complexo de ser obtido.

Considerando o método proposto, de correção estratificada (CE), pelo agrupamento com base no teste de Scott \& Knott (1974), obtiveram-se as informações constantes na Tabela 5. Em alguns ambientes, o teste de comparação de médias pelo método de agrupamento de Scott \& Knott não mostrou diferenças significativas entre as médias, apesar de as diferenças terem sido observadas pelo teste F. Nestas situações, optou-se por discriminar os grupos, adotando nível descritivo do teste até $12 \%$ de probabilidade, analisando cada partição dos grupos realizados. Assim, para as análises dos ambientes 1, 3, 4 e 6, os grupos foram estabelecidos, adotando os níveis 6,5 , $2,26,9,38$ e $11,70 \%$ de probabilidade, respectivamente.

Observa-se, portanto, inicialmente, comportamento diferenciado em cada ambiente para os estandes médios, número de cultivares e produção média por planta em cada grupo (Tabela 5). Isto mostra, em condições naturais de campo, uma relação freqüente entre o maior estande médio e a maior produção por

Tabela 5. Valores do estande médio, número de cultivares, produção média por planta, coeficiente de regressão residual estratificado $\left(b_{k}\right)$, e coeficiente de compensação por ausência de competição (â) de acordo com a partição de grupos pelo teste de Scott \& Knott (1974), em milho.

\begin{tabular}{cccclc}
\hline Ambiente & Estande médio & Número de cultivares & Produção média(g/planta) & $\mathrm{b}_{\mathrm{k}}(\mathrm{g} / \mathrm{planta})$ & $\hat{\mathrm{a}}$ \\
\hline 1 & $\mathrm{a}=48,69$ & 27 & 110,05 & $\mathrm{~b}_{\mathrm{a}}=12,79$ & 0,8838 \\
& $\mathrm{~b}=44,22$ & 6 & 104,05 & $\mathrm{~b}_{\mathrm{b}}=-19,83$ & 1,1906 \\
3 & $\mathrm{a}=48,96$ & 17 & 135,89 & $\mathrm{~b}_{\mathrm{a}}=129,08$ & 0,0501 \\
& $\mathrm{~b}=44,69$ & 12 & 136,74 & $\mathrm{~b}_{\mathrm{b}}=96,03$ & 0,2978 \\
& $\mathrm{c}=40,25$ & 4 & 113,58 & $\mathrm{~b}_{\mathrm{c}}=62,72$ & 0,4478 \\
4 & $\mathrm{a}=49,69$ & 32 & 133,23 & $\mathrm{~b}_{\mathrm{a}}=74,53$ & $-(1)$ \\
& $\mathrm{b}=42,33$ & 1 & 137,74 & $\mathrm{~b}_{\mathrm{a}}=124,86$ & 0,4406 \\
6 & $\mathrm{a}=48,67$ & 30 & 130,23 & $\mathrm{~b}_{\mathrm{b}}=60,60$ & 0,0412 \\
& $\mathrm{~b}=42,44$ & 3 & 124,93 & 0,5149 \\
\hline
\end{tabular}

(1)Indica a inexistência de valor, em razão da presença de uma só cultivar no grupo. 
planta. Além disso, demonstra, a princípio, que as cultivares com maior potencial de estande final possuem maior potencial genético para produção por planta, o que justifica o uso da correção estratificada.

Este potencial diferenciado, demonstrado pelos valores de $b_{\mathrm{k}}$ em cada ambiente, mostra que a produção média ( $\mathrm{g} / \mathrm{planta})$, acrescida por cada planta perdida será sempre maior no grupo de maior estande, e menor no de cultivares de menor estande. Isso demonstra, também, maior potencial produtivo das cultivares que compõem estes grupos. Por outro lado, os valores de â, obtidos com a estratificação, demonstram que apenas o grupo $b$ do ambiente 3 possui valor próximo ( $\hat{\mathrm{a}}=0,2978)$ ao preconizado por Zuber (1942).

\section{Conclusões}

1. A estatística $P_{i(100 / C V)}$ e $P_{i(F)}$ é eficiente para a interpretação conjunta dos ambientes na eficácia dos métodos de correção, considerando CV e F respectivamente, sendo de fácil interpretação.

2. A estatística $P_{\mathrm{mi}}$ é eficiente para a interpretação conjunta dos ambientes na eficácia dos métodos de correção, considerando simultaneamente os parâmetros $\mathrm{CV}$ e $\mathrm{F}$.

3. O melhor método de correção de parcelas é o de correção estratificada demonstrado pela estatística $\mathrm{P}_{\mathrm{mi}}$.

4. A maior eficácia do método de correção estratificada é demonstrada pela capacidade de correção mais adequada das cultivares, em que as mais produtivas são, também, as de maior potencial em deixar descendentes, de acordo com o valor do estande final por parcela.

5. O método proposto neste trabalho é eficiente em corrigir a produção de parcelas nos ambientes em que a estatística $\mathrm{F}$ tenha demonstrado diferença entre as cultivares em relação à capacidade de deixar descendentes, sendo, portanto, recomendado, tanto para correção em ambientes isolados, quanto na análise conjunta destes.

\section{Referências}

ÁVILA, A. V.; SÁNCHES, F. M. Comparación de métodos de ajuste para corrección por fallas en sorgos para grano. Agrociencia, Chapingo, v. 31, p. 45-64, 1978.
CARNEIRO, P. C. S. Novas metodologias de análise da adaptabilidade e estabilidade de comportamento. Viçosa : UFV, 1998. 168 p. Tese de Doutorado.

CRUZ, V. F. da. Estudo sobre a correção de produção de parcelas em ensaios com milho. Piracicaba : ESALQ, 1971. 143 p. Tese de Doutorado.

LIN, C. S.; BINNS, M. R. A superiority measure of cultivar performance for cultivar $\mathrm{x}$ location data. Canadian Journal of Plant Science, Ottawa, v. 68, n. 3, p. 193-198, 1988.

MORAIS, A. R.; OLIVEIRA, A. C.; CRUZ, J. C. Comparação de métodos de correção de produções de milho em parcelas experimentais. Relatório Técnico Anual do CNPMS: 1980-1984, Sete Lagoas, p. 130, 1986a.

MORAIS, A. R.; OLIVEIRA, A. C.; CRUZ, J. C. Correção de produções de grãos de milho em parcelas experimentais. Relatório Técnico Anual do CNPMS: 19801984, Sete Lagoas, p. 130-132, 1986 b.

SCHMILDT, E. R. Correção de rendimento de parcelas, estratificação ambiental e adaptabilidade e estabilidade de cultivares de milho. Viçosa : UFV, 2000. 110 p. Tese de Doutorado.

SCOTT, A. J.; KNOTT, M. A. A cluster analysis method for grouping means in the analysis of variance. Biometrics, Washington, v. 30, n. 3, p. 507-512, 1974.

STEEL, R. G. D.; TORRIE, J. H. Principles and procedures of statistics: a biometrical approach. 2. ed. New York: McGraw-Hill, 1980. 633 p.

VENCOVSKY, R.; CRUZ, C. D. Comparação de métodos de correção de rendimento de parcelas experimentais com estandes variados: I. Dados simulados. Pesquisa Agropecuária Brasileira, Brasília, v. 26, n. 5, p. 647-657, maio 1991.

VERONESI, J. A.; CRUZ, C. D.; CORRÊA, L. A.; SCAPIM, C. A. Comparação de métodos de ajuste do rendimento de parcelas com estandes variados. Pesquisa Agropecuária Brasileira, Brasília, v. 30, n. 2, p. 169-174, fev. 1995.

ZUBER, M. S. Relative efficiency of incomplete block designs using corn uniform trial data. Journal of the American Society of Agronomy, Madison, v. 34, n. 1, p. 30-47, 1942. 